\title{
Determination of Electron Transfer Number for Oxygen Reduction Reaction: from Theory to Experiment
}

\author{
Ruifeng Zhou ${ }^{1,2}$, Yao Zheng ${ }^{1}$, Mietek Jaroniec ${ }^{3}$ and Shi-Zhang Qiao ${ }^{1, *}$ \\ ${ }^{1}$ School of Chemical Engineering, the University of Adelaide, Adelaide, SA 5005, Australia. \\ ${ }^{2}$ Australian Institute for Bioengineering and Nanotechnology, the University of Queensland, \\ St Lucia, QLD 4072, Australia. \\ ${ }^{3}$ Department of Chemistry and Biochemistry, Kent State University, Kent, OH 44242, United \\ States. \\ *E-mail: s.qiao@adelaide.edu.au
}

\section{Experimental and Calculation Details}

\subsection{Preparation of catalysts}

N-MCN: In a typical synthesis of N-MCN, a solution was prepared by mixing $80 \mathrm{~mL}$ of ethanol and $200 \mathrm{~mL}$ of distilled water. Subsequently, $1 \mathrm{~g}$ of F127, $1.3 \mathrm{~g}$ of CTAB, and $2 \mathrm{~g}$ of cysteine were added into the mixed solution under continuous stirring. Then, $2 \mathrm{~g}$ of 3 aminophenol was added and stirred until it was completely dissolved. Next, $2.8 \mathrm{~mL}$ of $37 \mathrm{wt} \%$ formaldehyde was dropped in and the stirring was continued for another $24 \mathrm{~h}$ at $25^{\circ} \mathrm{C}$. Finally, the mixture was transferred to an autoclave and kept at $100{ }^{\circ} \mathrm{C}$ for another $24 \mathrm{~h}$. The resulting resin was obtained by washing with water and ethanol for 3 times. In order to obtain N-MCN, the resin were carbonized under $\mathrm{N}_{2}$ flow in the tube furnace by heating up to $350{ }^{\circ} \mathrm{C}$ with a rate of $1{ }^{\circ} \mathrm{C} / \mathrm{min}$, dwelling of $2 \mathrm{~h}$, then heating up to $700{ }^{\circ} \mathrm{C}$ with a rate of $1{ }^{\circ} \mathrm{C} / \mathrm{min}$ and dwelling for $4 \mathrm{~h}$.

N-rGO: Graphite flake (3 g) was mixed with concentrated $\mathrm{H}_{2} \mathrm{SO}_{4}(12 \mathrm{~mL}), \mathrm{K}_{2} \mathrm{~S}_{2} \mathrm{O}_{8}(2.5 \mathrm{~g})$, and $\mathrm{P}_{2} \mathrm{O}_{5}(2.5 \mathrm{~g})$. The mixture was kept at $90{ }^{\circ} \mathrm{C}$ for $6 \mathrm{~h}$, then cooled to room temperature and diluted with $0.5 \mathrm{~L}$ of DI water. The suspension was filtered and washed with DI water. The solid was dried in vacuum at $50{ }^{\circ} \mathrm{C}$ overnight. The pre-treated graphite was put into cold $\left(0{ }^{\circ} \mathrm{C}\right)$ concentrated $\mathrm{H}_{2} \mathrm{SO}_{4}(120 \mathrm{~mL})$. Then, $\mathrm{KMnO}_{4}(15 \mathrm{~g})$ was added gradually under stirring and 
the temperature of the mixture was kept to be below $20{ }^{\circ} \mathrm{C}$ by ice cooling. The mixture was stirred at $40{ }^{\circ} \mathrm{C}$ for $3 \mathrm{~h}$, and then diluted with DI water $(250 \mathrm{~mL})$ using ice bath cooling. It was then stirred for $2 \mathrm{~h}$, and additional $0.7 \mathrm{~L}$ of DI water was added. $20 \mathrm{~mL}$ of $30 \% \mathrm{H}_{2} \mathrm{O}_{2}$ was added to the mixture. Then it was washed with 1:10 $\mathrm{HCl}$ aqueous solution $(1 \mathrm{~L})$. The resulting graphite oxide was diluted in DI water. Exfoliation was carried out by sonicating graphite oxide dispersion under ambient conditions for $30 \mathrm{~min}$, followed by centrifugation at $3000 \mathrm{rpm}$ for $30 \mathrm{~min}$ to eliminate non-exfoliated graphite.

To synthesize $\mathrm{N}-\mathrm{rGO}, 5 \mathrm{~mL}$ of $25 \% \mathrm{NH}_{3}$ aqueous solution was added into $65 \mathrm{~mL}$ of $0.1 \% \mathrm{GO}$ solution. The solution was stirred at $90{ }^{\circ} \mathrm{C}$ for $12 \mathrm{~h}$. After reduction, N-rGO was filtered and washed with water and dispersed in water by supersonication.

\subsection{Preparation of electrodes}

N-MCN: $2 \mathrm{mg}$ of N-MCN was dispersed in $0.2 \%$ Nafion aqueous solution by supersonication. $25 \mu \mathrm{L}$ of the catalyst ink was spread on the disk electrode and dried under ambient environment.

e-rGO: $25 \mu \mathrm{L}$ of $0.1 \%$ GO aqueous suspension and $25 \mu \mathrm{L}$ of $0.1 \%$ Nafion aqueous solution were spread on the disk electrode and dried under ambient environment. CV from $-0.2 \mathrm{~V}$ to 1 $\mathrm{V}$ was applied to the electrode until GO was fully reduced (no significant cathodic current was observed).

N-rGO: $25 \mu \mathrm{L}$ of $0.1 \% \mathrm{~N}-\mathrm{rGO}$ aqueous suspension and $25 \mu \mathrm{L}$ of $0.1 \%$ Nafion aqueous solution were spread on the disk electrode and dried under ambient environment.

$\mathbf{C o}(\mathrm{OH})_{2} / \mathrm{N}-\mathbf{r G O}: 25 \mu \mathrm{L}$ of $0.1 \mathrm{M} \mathrm{CoCl}_{2}$ was spread on the N-rGO electrode. The electrode was kept in water saturated atmosphere for 1 hour. The drop of $\operatorname{Co}(\mathrm{Ac})_{2}$ was then flushed with DI water and the electrode was dried under ambient environment.

Au: A disk as the working electrode was immersed in $0.025 \mathrm{M} \mathrm{HAuCl}_{4}$ solution. A Pt wire was used as a counter electrode. A constant current of $-2.5 \mathrm{~mA} \mathrm{~cm}{ }^{-2}$ was applied to the electrode for $100 \mathrm{~s}$. The electrode was then washed with DI water.

Ag: A disk as the working electrode was immersed in $0.1 \mathrm{M} \mathrm{AgNO}_{3}$ solution. A Pt wire was used as a counter electrode. A constant current of $-2.5 \mathrm{~mA} \mathrm{~cm}{ }^{-2}$ was applied to the electrode for $100 \mathrm{~s}$. The electrode was then washed with DI water. 
Ru: A disk as the working electrode was immersed in $0.05 \mathrm{M} \mathrm{RuCl}_{3}$ solution. A Pt wire was used as a counter electrode. A constant current of $-2.5 \mathrm{~mA} \mathrm{~cm}^{-2}$ was applied to the electrode for $100 \mathrm{~s}$. The electrode was then washed with DI water.

\subsection{Calibration of $\mathrm{N}_{\mathrm{C}}$}

When calibrating $\mathrm{N}_{\mathrm{C}}, \mathrm{N}_{2}$ saturated $0.1 \mathrm{M} \mathrm{KOH}$ and $0.004 \mathrm{M} \mathrm{K} \mathrm{K}_{3} \mathrm{Fe}(\mathrm{CN})_{6}$ were used as the electrolyte. The electrodes were the same as those used in the ORR measurements. The electrode was rotated at the certain angular velocity and the amperometric i-t measurements were performed. The voltage of the disk and ring were set to $0.1 \mathrm{~V}$ and $1.5 \mathrm{~V}$, respectively. The reactions taking place on the disk and ring are:

Disk: $\mathrm{Fe}(\mathrm{CN})_{6}{ }^{3-}+e^{-} \rightarrow \mathrm{Fe}(\mathrm{CN})_{6}{ }^{4-}$

Ring: $\mathrm{Fe}(\mathrm{CN})_{6}{ }^{4-} \rightarrow \mathrm{Fe}(\mathrm{CN})_{6}{ }^{3-}+e^{-}$

The measurement lasted $60 \mathrm{~s}$ and the disk and ring currents $\left(I_{d}\right.$ and $\left.I_{r}\right)$ at the last $10 \mathrm{~s}$ were averaged. The measurement was repeated once with the disk disconnected. The ring current $\left(\mathrm{I}_{\mathrm{r} 0}\right)$ at the last $10 \mathrm{~s}$ was averaged. $\mathrm{I}_{\mathrm{r} 0}$ includes all the anodic currents that are not from $\mathrm{Fe}(\mathrm{CN})_{6}{ }^{4-}$ reduced on the disk. They may be related to the oxidation of water, free $\mathrm{Fe}(\mathrm{CN})_{6}{ }^{4-}$ or any impurities. The $\mathrm{N}_{C}$ value is calculated by using the following equation:

$$
N_{C}=\frac{I_{r}-I_{r 0}}{I_{d}}
$$

\subsection{ORR measurements}

First, the electrolyte resistance was measured by electrochemical impedance spectroscopy (EIS). The Nyquist plots obtained for GC, Ag and N-rGO at $0.9 \mathrm{~V}$ vs RHE are shown below. At high frequency limit, the impedance is equal to the electrolyte resistance, which is about $30 \Omega$. This resistance was used in all further experiments. 


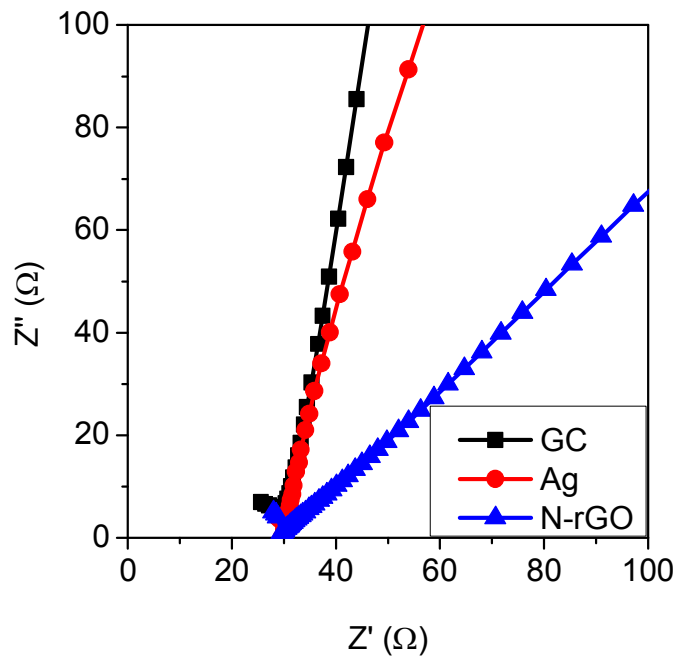

Then, the blank LSV curve was measured from $1 \mathrm{~V}$ to 0 with scanning rate of $10 \mathrm{mV} \mathrm{s}^{-1}$ in $\mathrm{N}_{2}$-saturated electrolyte. Next, the LSV curve was measured for ORR using the same electrode and electrolyte but saturated with $\mathrm{O}_{2}$. An example of the blank and ORR LSV curves and their difference is shown below for Ru catalyst.

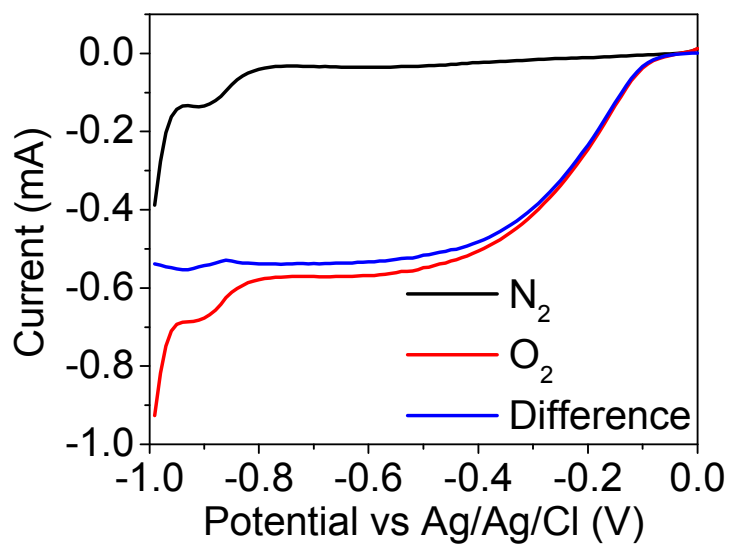

Figure S1. LSV curves for the $\mathrm{Ru}$ disk in $\mathrm{N}_{2}$ and $\mathrm{O}_{2}$ saturated $0.1 \mathrm{M} \mathrm{KOH}$ and their difference at $400 \mathrm{rpm}$.

\subsection{Derivation of equations (1) and (2)}

The total number of oxygen molecules, which participate in ORR can be calculated as follows:

$$
N_{O 2}=\frac{N_{A} \Delta t}{F}\left(\frac{i_{4}}{4}+\frac{i_{2}}{2}\right)
$$


where $\Delta t$ is the time interval; $N_{A}$ is the Avogadro constant; $\mathrm{F}$ is the Faraday constant; $i_{4}$ and $i_{2}$ are the currents of reactions (4e) and (2e). The total number of electrons, which are transferred, can be determined by the following equation:

$$
N_{e}=\frac{N_{A} \Delta t}{F}\left(i_{4}+i_{2}+i_{2},\right)
$$

where $i_{2}$, is the current of reaction (2'e). So, $\mathrm{n}$ can be expressed as follows:

$$
n=\frac{N_{e}}{N_{O 2}}=4 \times \frac{i_{4}+i_{2}+i_{2 \prime}}{i_{4}+2 i_{2}}
$$

The relationships between the disk and ring currents $\left\{i_{d}, i_{r}\right\}$ and $\left\{i_{4}, i_{2}, i_{2}\right\}$ are:

$$
\begin{gathered}
i_{d}=i_{4}+i_{2}+i_{2 \prime} \\
\frac{i_{r}}{N_{C}}=i_{2}-i_{2 \prime}
\end{gathered}
$$

So, substitution of $\left\{i_{4}, i_{2}, i_{2},\right\}$ with $\left\{i_{d}, i_{r}\right\}$ gives:

$$
n=4 \times \frac{i_{d}}{i_{d}+\frac{i_{r}}{N_{C}}}
$$

Similarly, the total number of oxygen molecules, which are reduced into hydrogen peroxide is:

$$
N_{H 2 O 2}=\frac{N_{A} \Delta t}{F}\left(\frac{i_{2}}{2}-\frac{i_{2 \prime}}{2}\right)
$$

So:

$$
p=\frac{N_{H 2 O 2}}{N_{O 2}}=2 \times \frac{\frac{i_{r}}{N_{C}}}{i_{d}+\frac{i_{r}}{N_{C}}}
$$

\subsection{Parameters used in the KL method}

$\mathrm{F}=96485 \mathrm{C}$

$\mathrm{C}_{\mathrm{O}}{ }^{*}=1.22 \mathrm{~m}^{-3}$

$\mathrm{D}_{\mathrm{O}}=1.98 \cdot 10^{-9} \mathrm{~m}^{2} \mathrm{~s}^{-1}$

$v=1.004 \cdot 10^{-6} \mathrm{~m}^{2} \mathrm{~s}^{-1}$

$\mathrm{D}_{\mathrm{P}}=1.27 \cdot 10^{-9} \mathrm{~m}^{2} \mathrm{~s}^{-1}$ 


\subsection{Measurement errors in the KL method}

Beside the $\mathrm{N}_{\mathrm{C}}$ and ring reaction, many other factors can cause errors in electrochemical measurements, which result in incorrect $\mathrm{n}$ values. The resistance and capacitance can influence the measured potential and current density but they can be corrected easily by IR compensation and background subtraction. However, the instability of system and the change of geometry are inevitable. First, the ORR current is not extremely stable on any catalyst. Even if the change of activity is too small to be observed by naked eye, it may have significant impact on the regression analysis in the KL method when current is very small, near onset potential. Second, $\mathrm{N}_{\mathrm{C}}$ has been proved to be affected by the catalyst layer due to the change in geometry. The KL method uses the same hydrodynamic model so it should be affected too. To evaluate this impact, the reduction of $\mathrm{Fe}(\mathrm{CN})_{6}{ }^{3-}$ is used again, in which $\mathrm{n}$ is always 1 . Therefore, the KL plot must be linear, and all the data points must coincide at same $\omega$, if the reaction is mass transfer limited. Any inconsistent data can be attributed to the change of hydrodynamics.

The $\mathrm{KL}$ plot of $\mathrm{Fe}(\mathrm{CN})_{6}{ }^{3-}$ reduction on a bare $\mathrm{RDE}$ at $0.1 \mathrm{~V}$ is shown in Figure S2a. As expected, the KL plot shows extremely good linearity with correlation coefficient (r) as high as 0.999998 . 

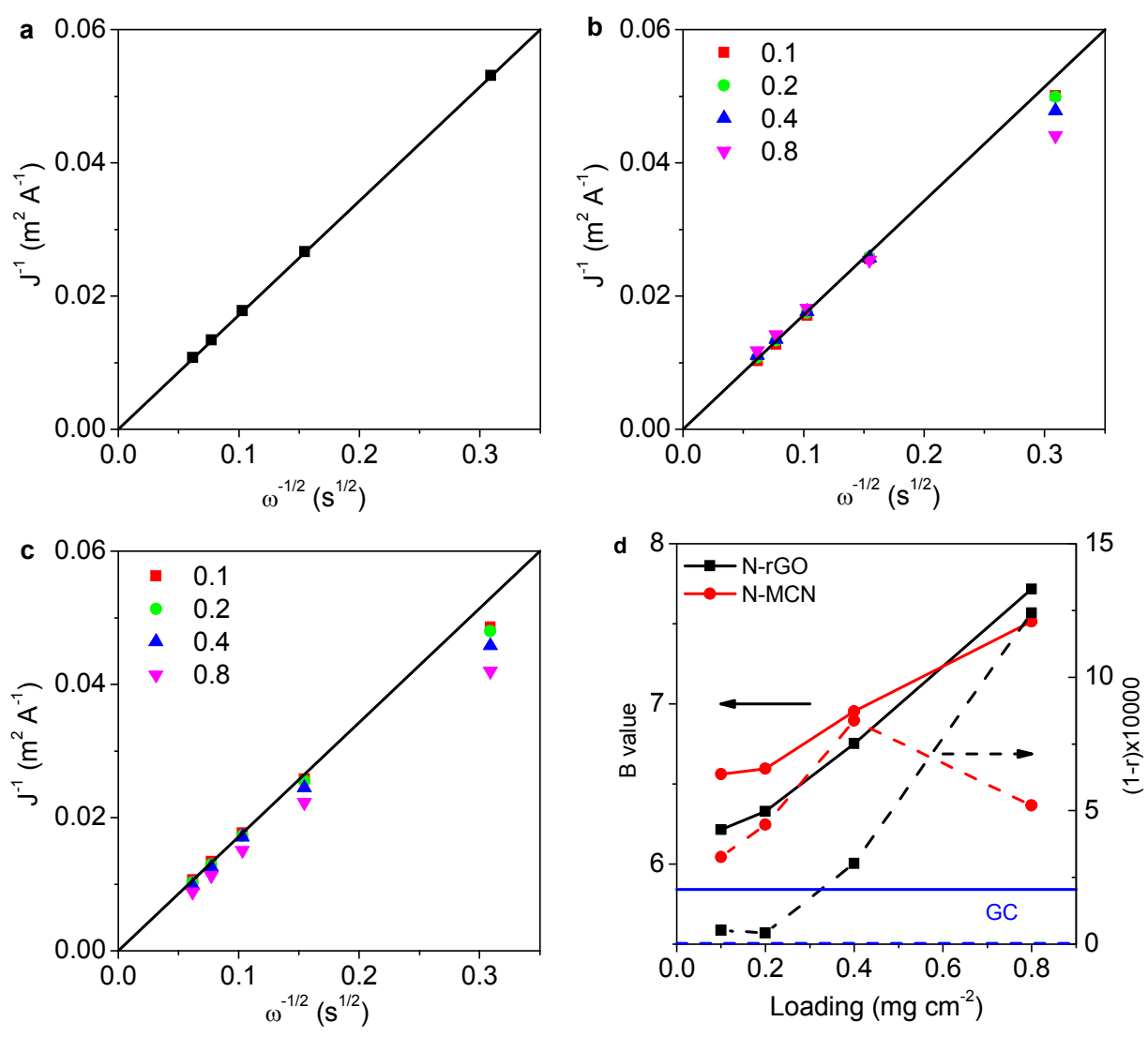

Figure S2. KL plot of $\mathrm{Fe}(\mathrm{CN})_{6}{ }^{3-}$ on bare (a), N-rGO (b) or N-MCN (c) loaded RDE. The potential is $0.1 \mathrm{~V}$. The numbers beside the line are $\omega$ in rpm and the numbers in legend are the loading amounts of catalysts in $\mathrm{mg} \mathrm{cm}^{-2}$. (d) B and $\mathrm{r}$ values of the corresponding KL plot.

The fitted line passes the origin, showing very large $J_{K}$ so the apparent current is completely limited by mass transfer. This proves that the KL theory is suitable and accurate for $\mathrm{Fe}(\mathrm{CN})_{6}{ }^{3-}$ reduction on an ideal RDE. Afterwards, the KL plots were measured on N-rGO and N-MCN loaded RDE under same conditions. As shown in Figures S2b and S2c, the data points do not appear on the fitted line of bare RDE. The deviation increases with increasing loading of catalysts, which is caused by the previously mentioned change in the electrode geometry. In this case, if the KL plots are fitted, the B value in equation (4) is quite different from that of bare RDE (Figure S2d). On bare RDE, B is 5.84, while on RDE loaded with NrGO or N-MCN, it increases from about 6.2 to 7.7 as the loading increases. As a result, if $\mathrm{n}$ is calculated from the KL plot, an error from $6 \%$ to $30 \%$ can be induced. The $\mathrm{r}$ of the KL plot obtained for N-rGO and N-MCN loaded RDE range from 0.99995 to 0.9988 . For naked eye, these plots are linear, but actually much poorer than that on bare RDE. This proves that $\mathrm{n}_{\mathrm{KL}}$ may not be accurate even if the KL plot "looks linear". In our measurement of $\mathrm{n}_{\mathrm{KL}}$ for ORR in 
the next section, the lowest catalyst loading is applied so the possibly most accurate results are shown. 


\section{Supporting Figures}

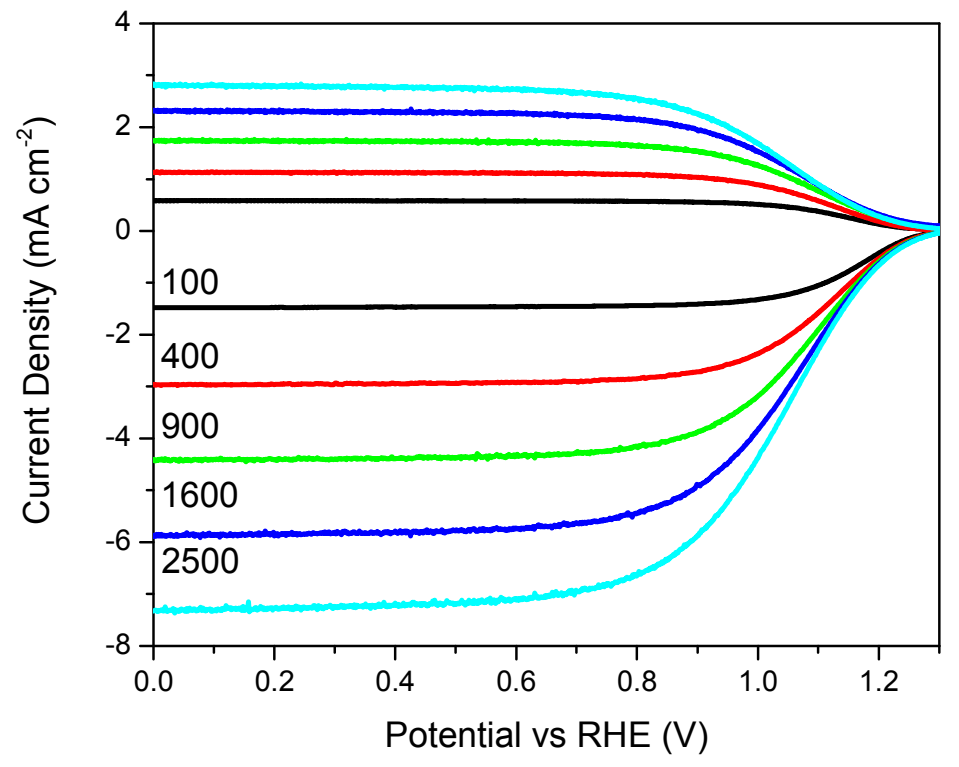

Figure S3. LSV curves for a bare GC disk in $0.1 \mathrm{M} \mathrm{KOH}$ and $0.004 \mathrm{M} \mathrm{K}_{3} \mathrm{Fe}(\mathrm{CN})_{6}$. The ring is biased at $1.5 \mathrm{~V}$. The cathodic current is the disk current and the anodic current is the ring current. The current is normalized by taking into account the disk area. The numbers at the curves are the angular velocities in rpm.

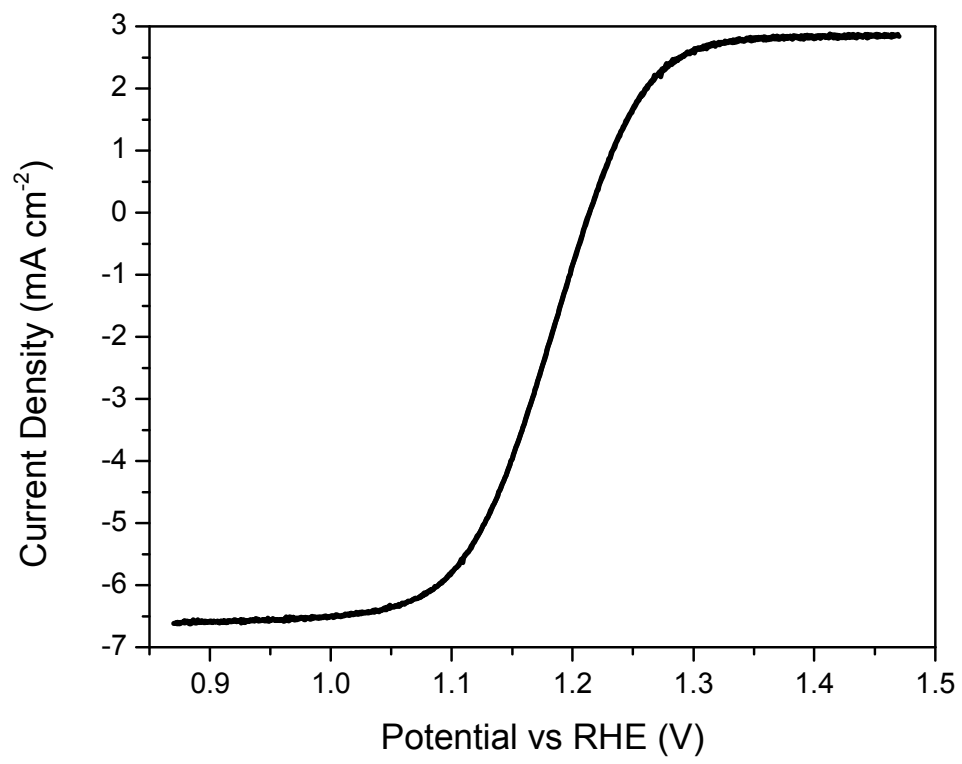

Figure S4. LSV curve for Pt ring in $0.1 \mathrm{M} \mathrm{KOH}$ and $0.004 \mathrm{M} \mathrm{K}_{3} \mathrm{Fe}(\mathrm{CN})_{6}$. The disk is a bare GC and biased at $0.1 \mathrm{~V}$. The current is normalized by taking into account the disk area. 


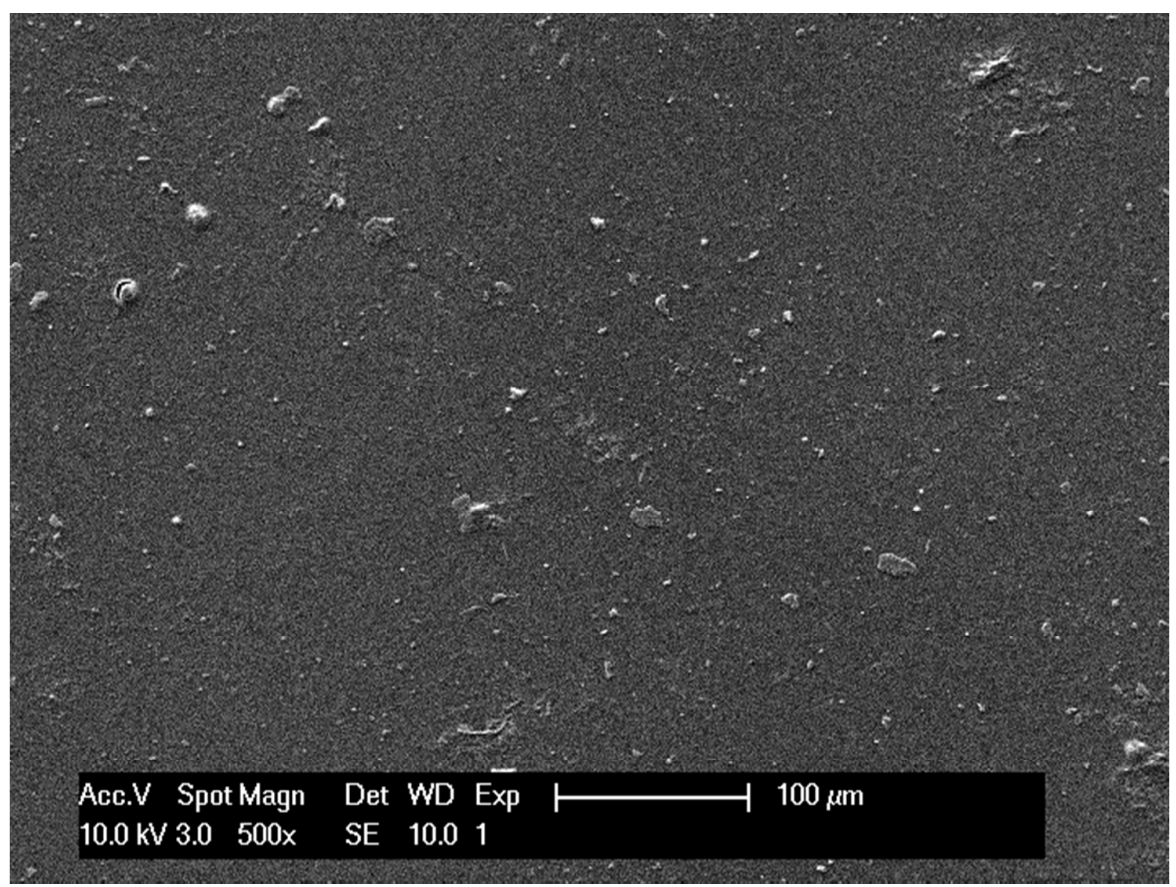

Figure S5. SEM image of the surface of N-rGO electrode.

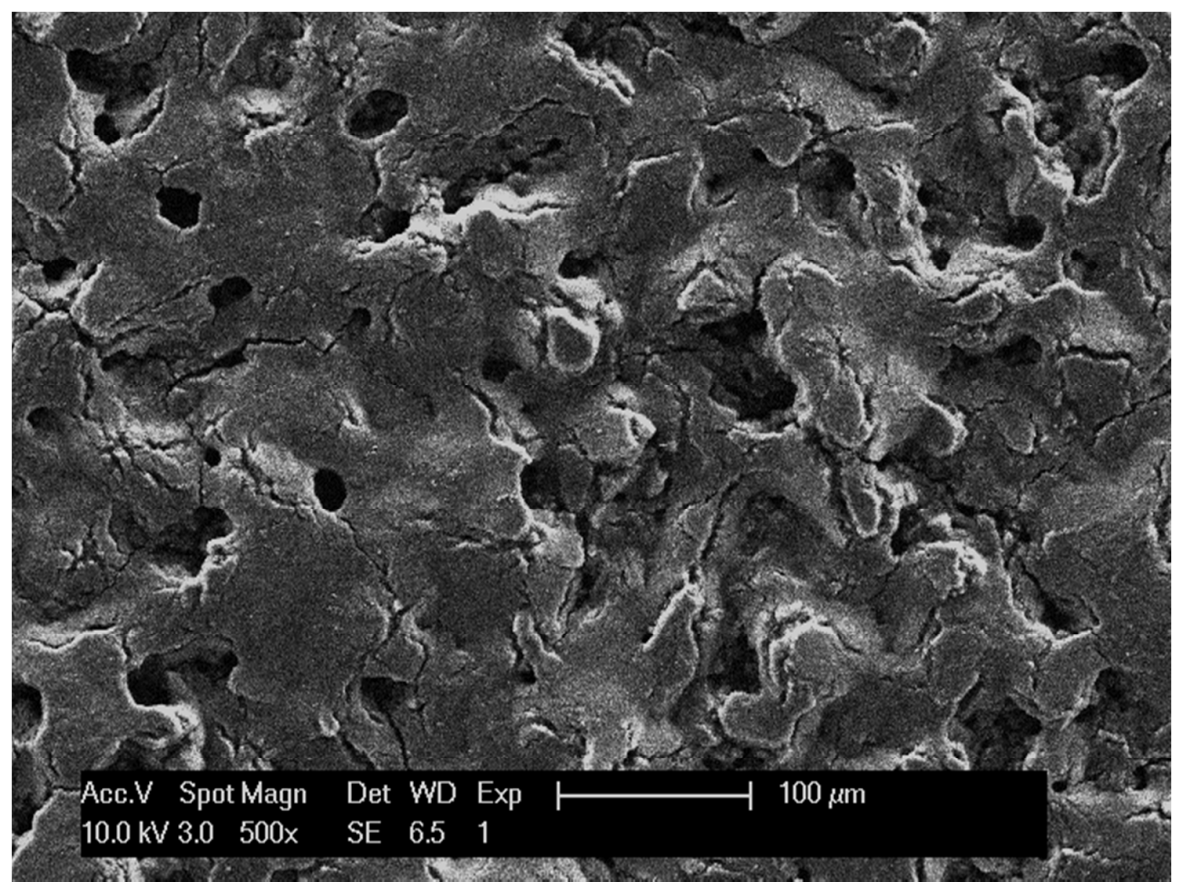

Figure S6. SEM image of the surface of N-MCN electrode. 


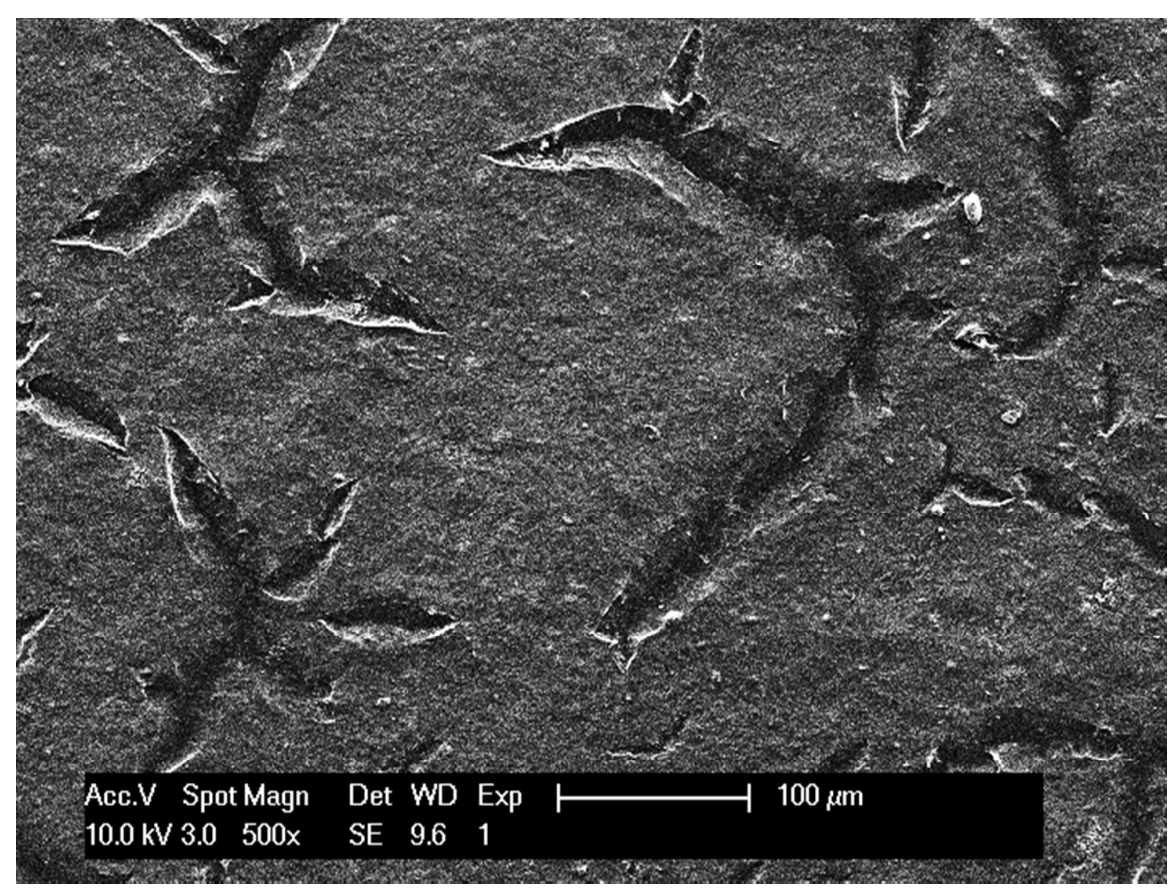

Figure S7. SEM image of the surface of $\mathrm{Co}(\mathrm{OH})_{2} / \mathrm{N}-\mathrm{rGO}$ electrode.
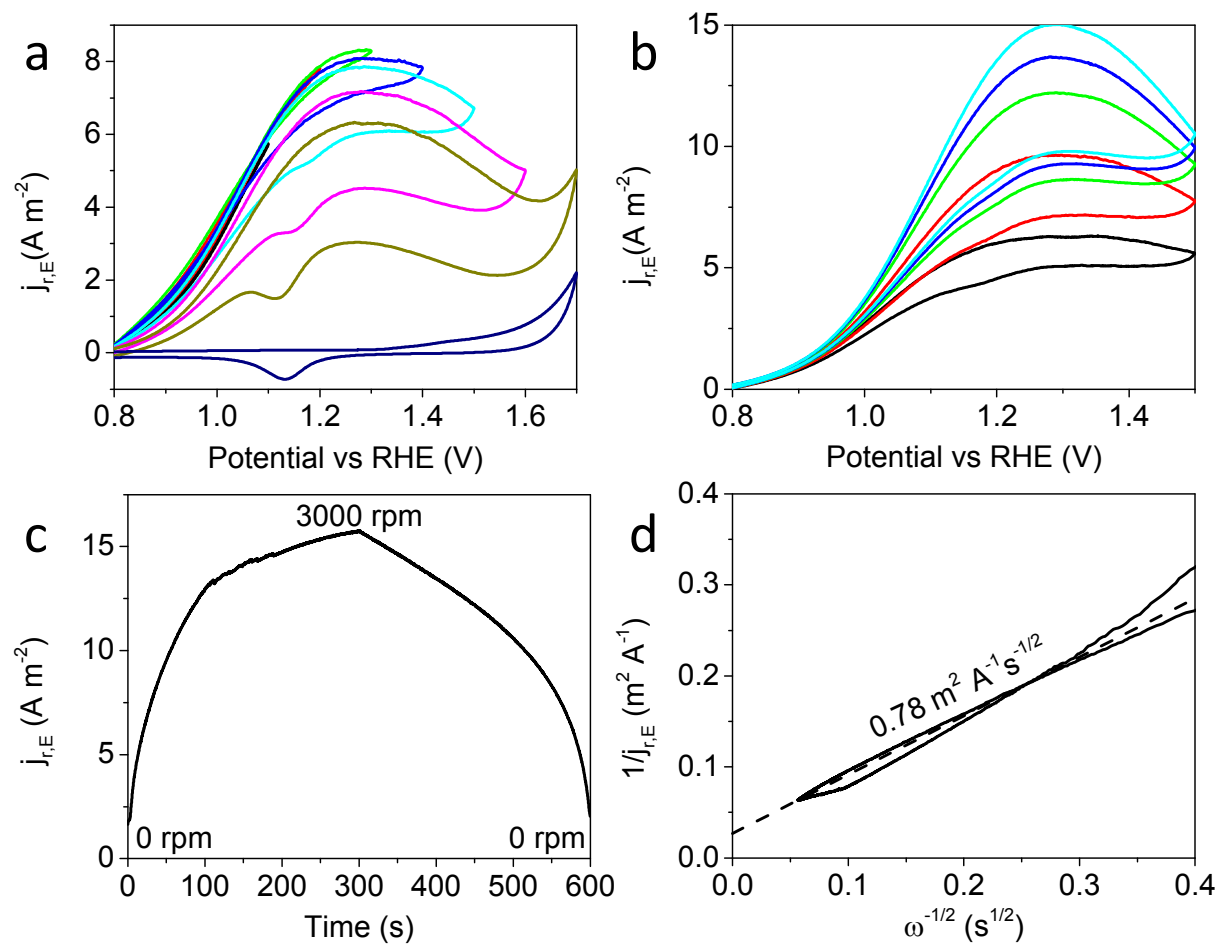

Figure S8. $\mathrm{H}_{2} \mathrm{O}_{2}$ oxidation experiment in acidic electrolyte on Pt ring. (a) $\mathrm{CV}$ at $400 \mathrm{rpm}$. (b) $\mathrm{CV}$ at $\omega=225 \mathrm{rpm}, 625 \mathrm{rpm}, 1225 \mathrm{rpm}, 2025 \mathrm{rpm}$ and $3025 \mathrm{rpm}$ from bottom to top. The scan rate is $10 \mathrm{mV} \mathrm{s}^{-1}$. (c) The amperometric i-t plots at $1.3 \mathrm{~V}$. (d) $\mathrm{KL}$ plot derived from (e). The electrolyte is $0.05 \mathrm{M} \mathrm{H}_{2} \mathrm{SO}_{4}$ containing $1 \mathrm{mM} \mathrm{H}_{2} \mathrm{O}_{2}$ for all experiments except the lowest curves in panel (a) (without $\mathrm{H}_{2} \mathrm{O}_{2}$ ). 


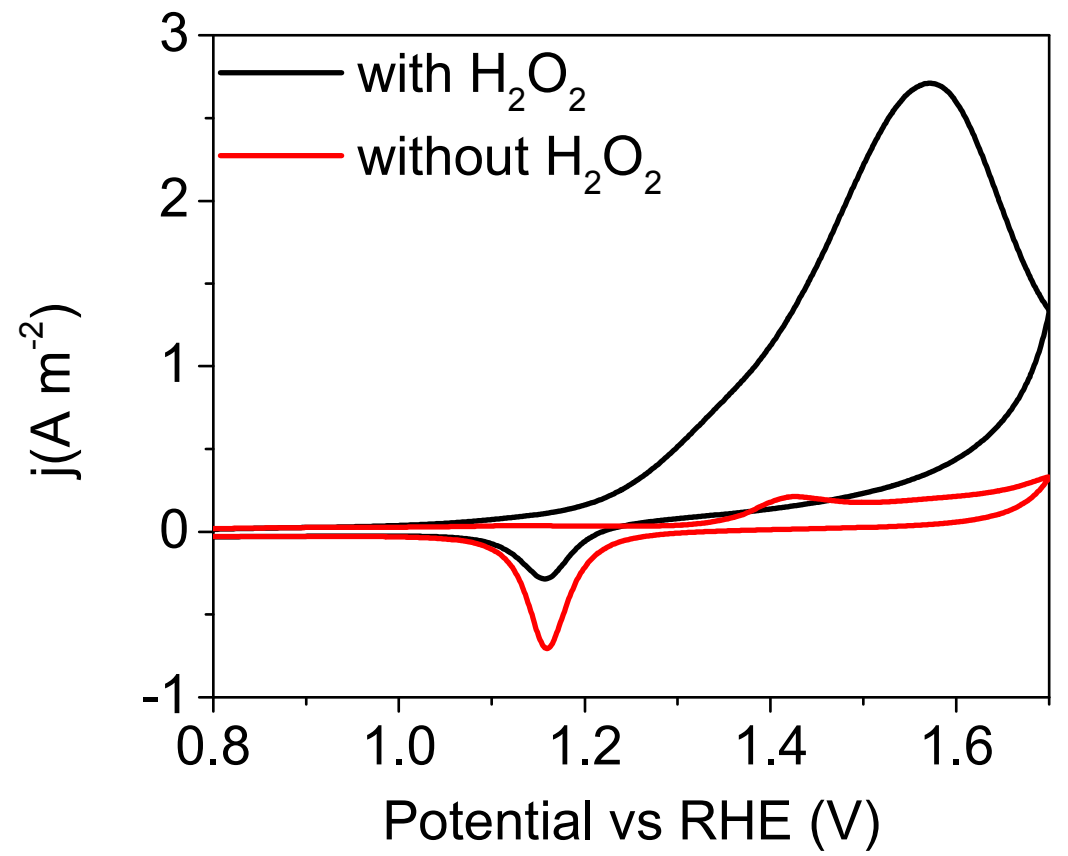

Figure S9. CV curve for $\mathrm{Au}$ in $0.05 \mathrm{M} \mathrm{H}_{2} \mathrm{SO}_{4}$ at $400 \mathrm{rpm}$. The scan rate is $10 \mathrm{mV} \mathrm{s}^{-1}$.

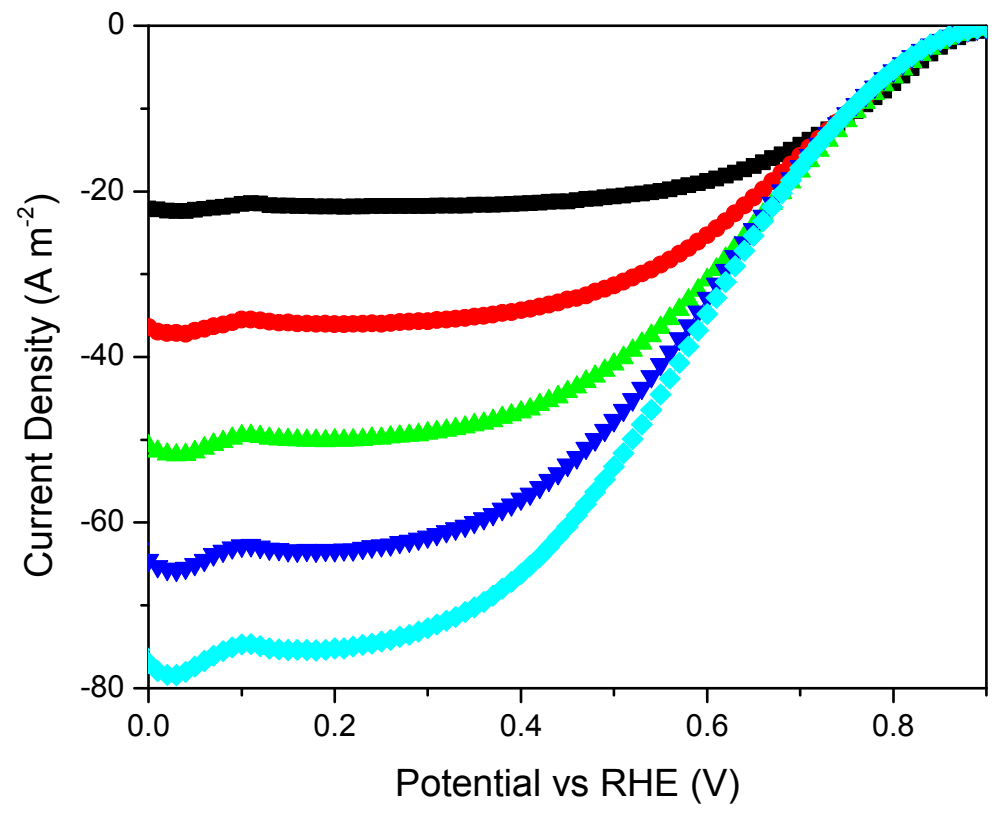

Figure S10. LSV curves for the Ru disk. The angular velocities are $225 \mathrm{rpm}$ (Black), $625 \mathrm{rpm}$ (red), 1225 rpm (green) 2025 rpm (blue) and 3025 rpm (cyan). 


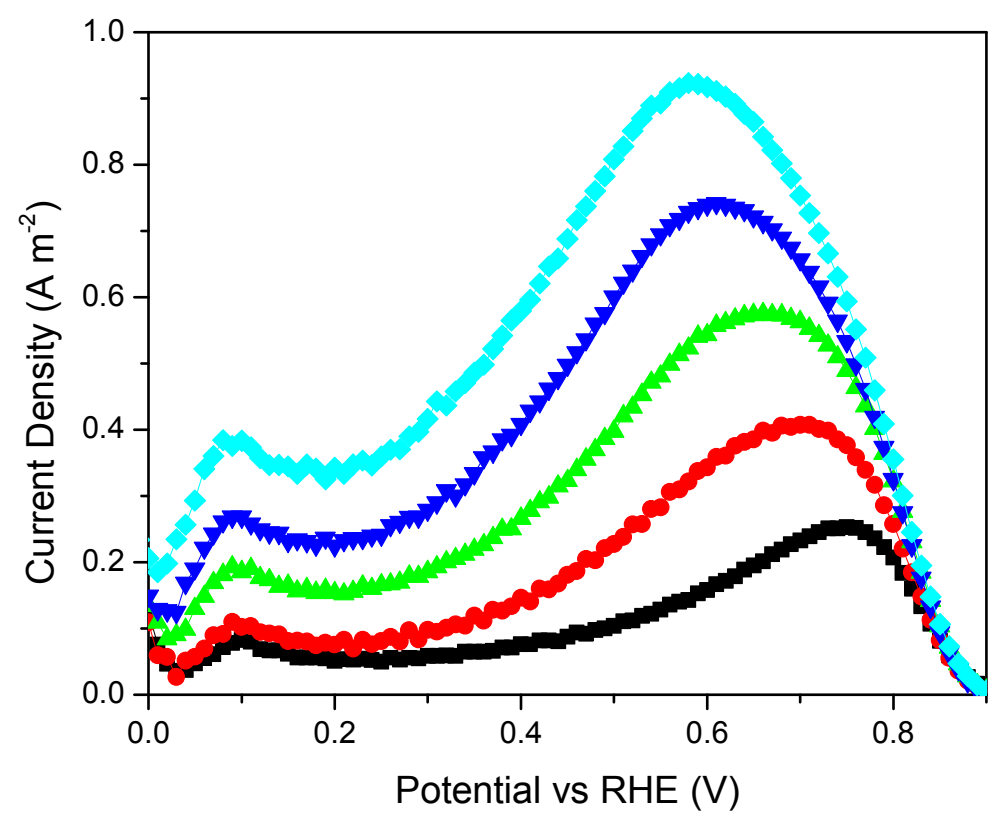

Figure S11. Ring current densities of Ru.

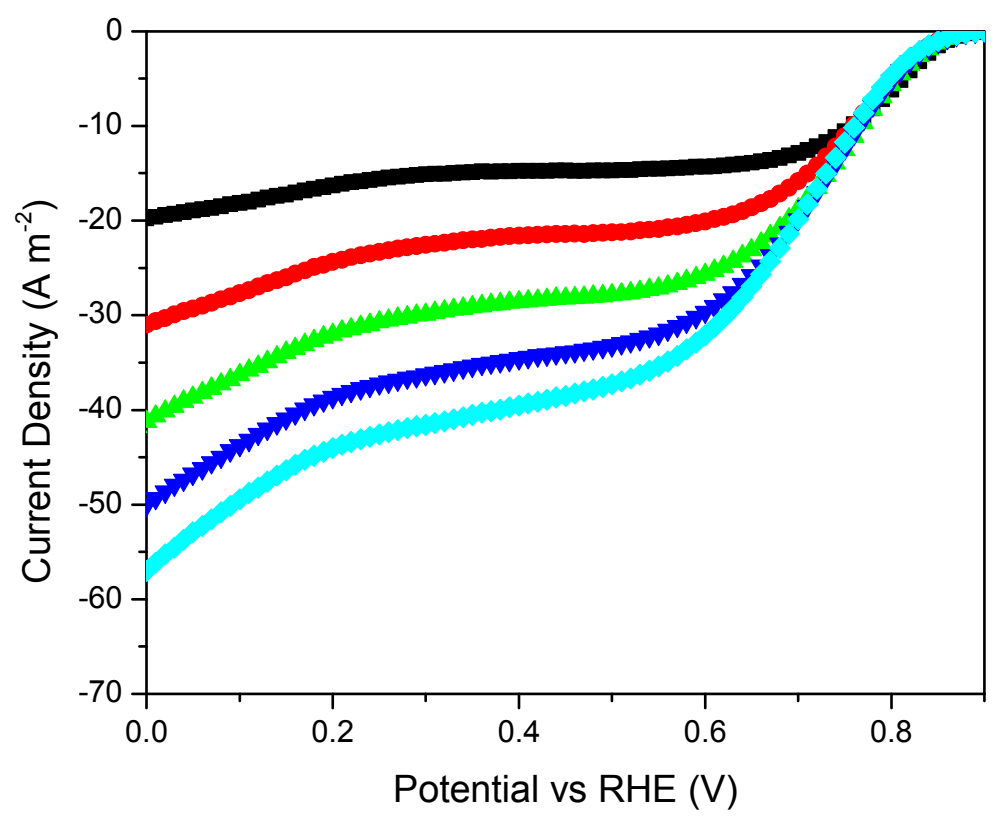

Figure S12. LSV curves for the Au disk. 


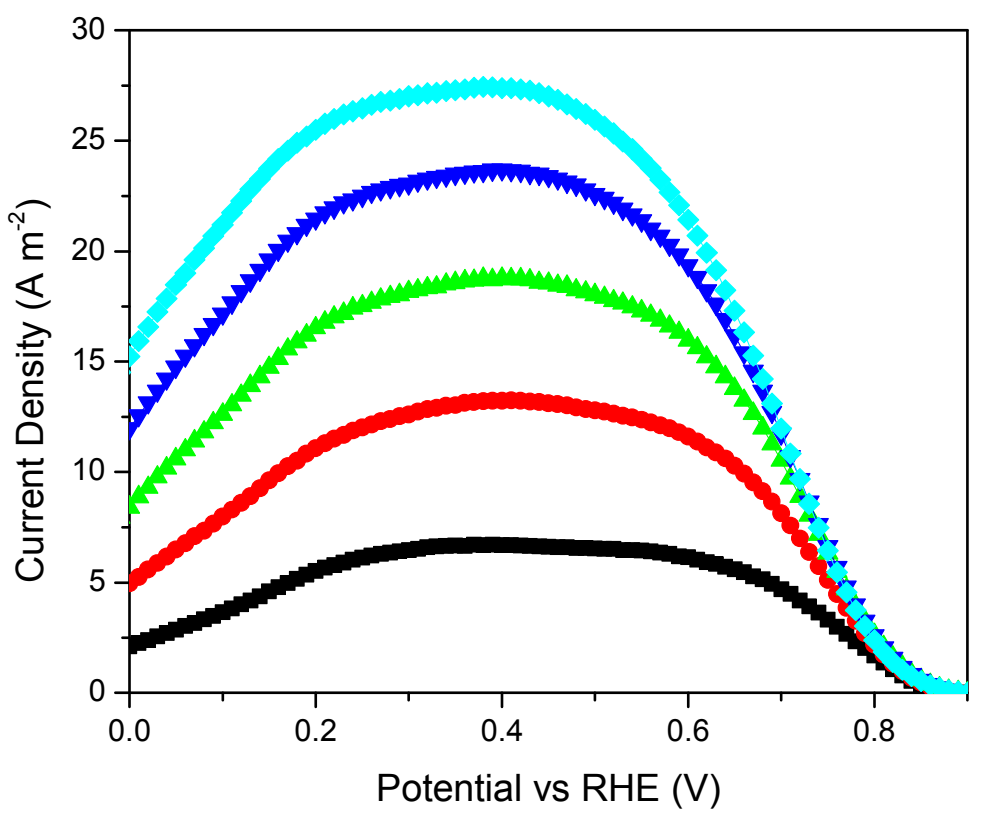

Figure S13. Ring current densities at the Au electrode.

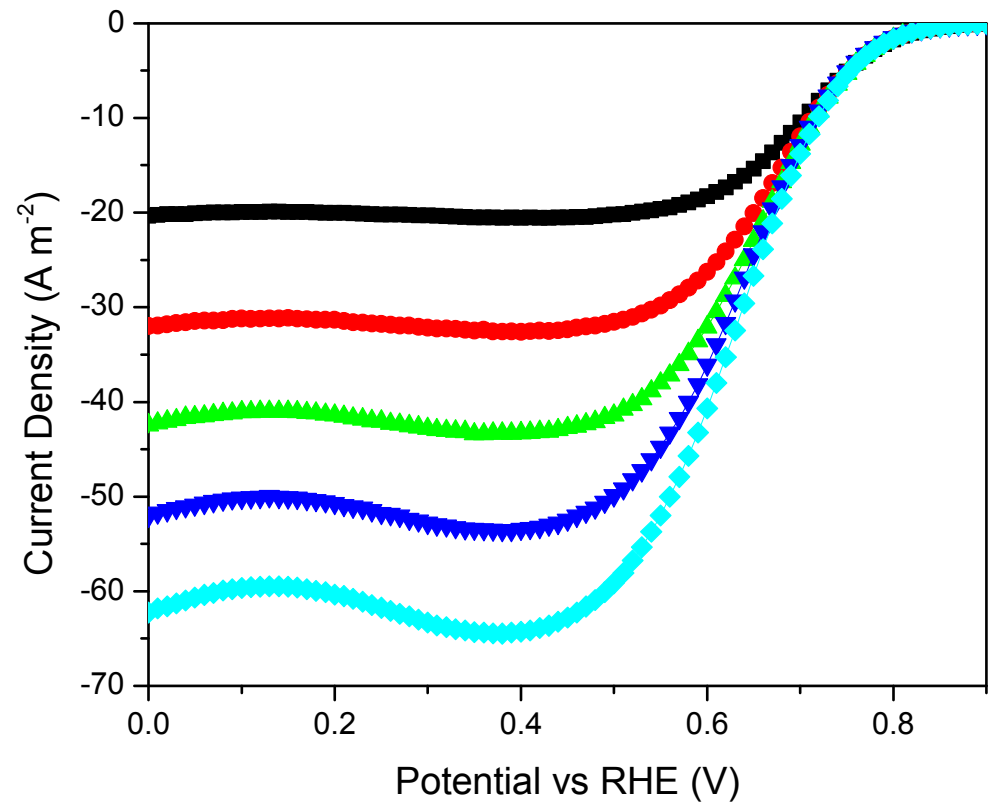

Figure S14. LSV curves for the Ag disk. 


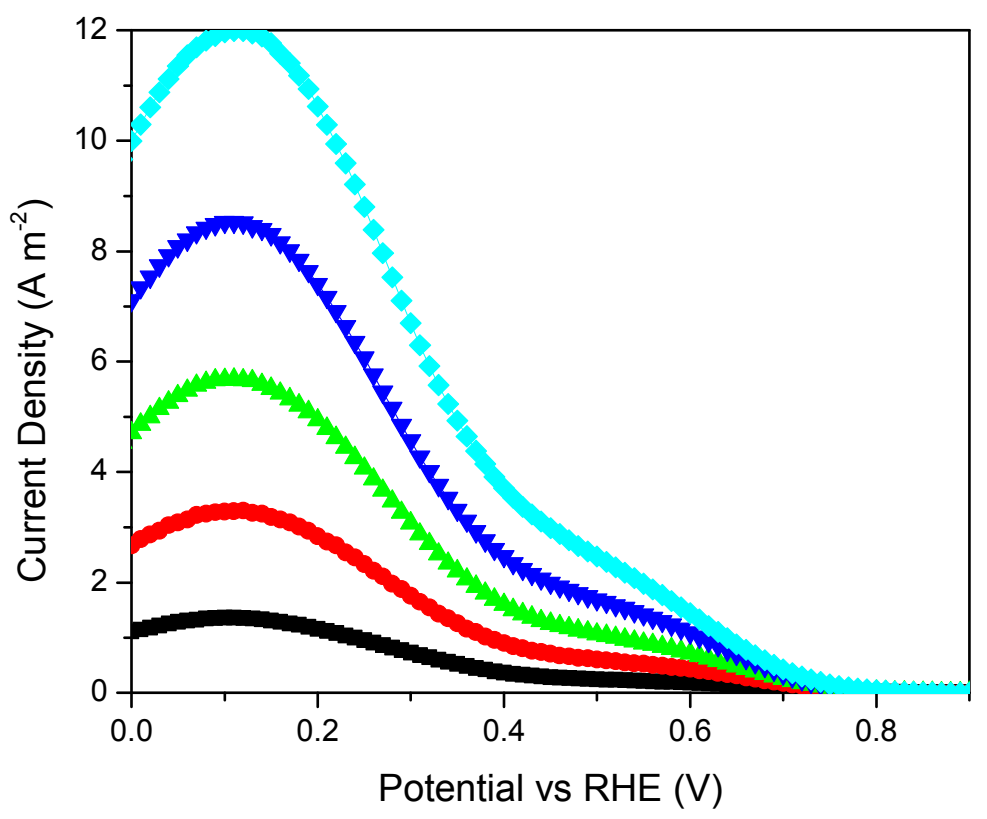

Figure S15. Ring current densities at the Ag electrode.

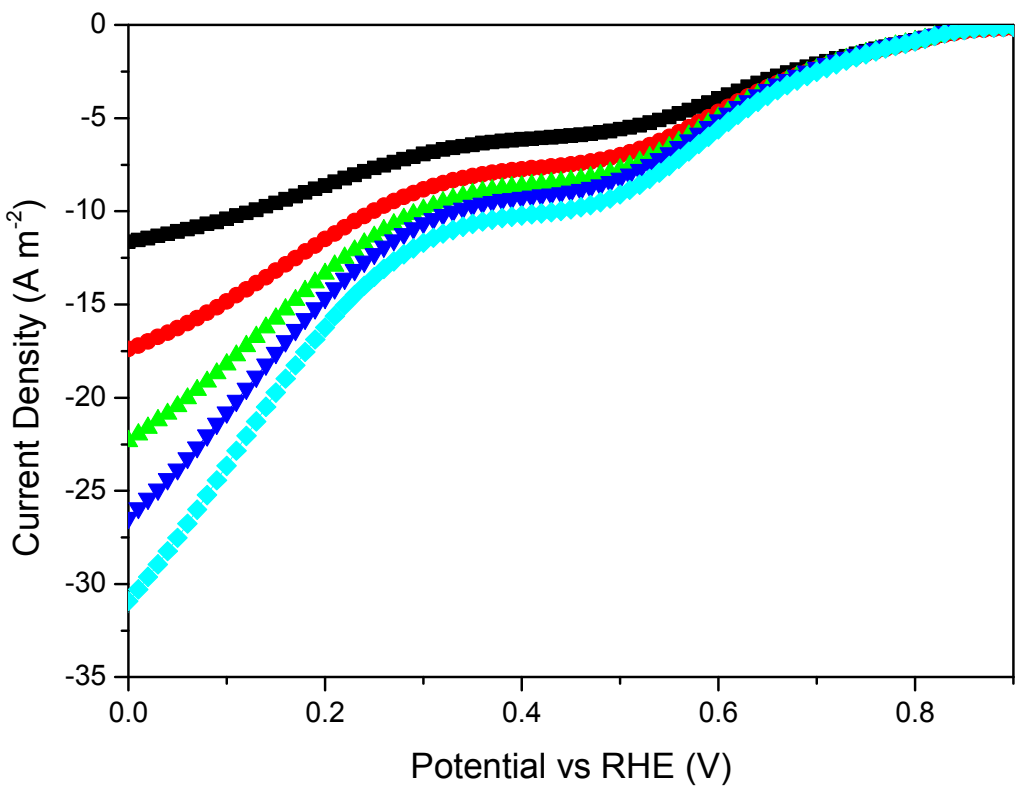

Figure S16. LSV curves for the e-rGO disk. 


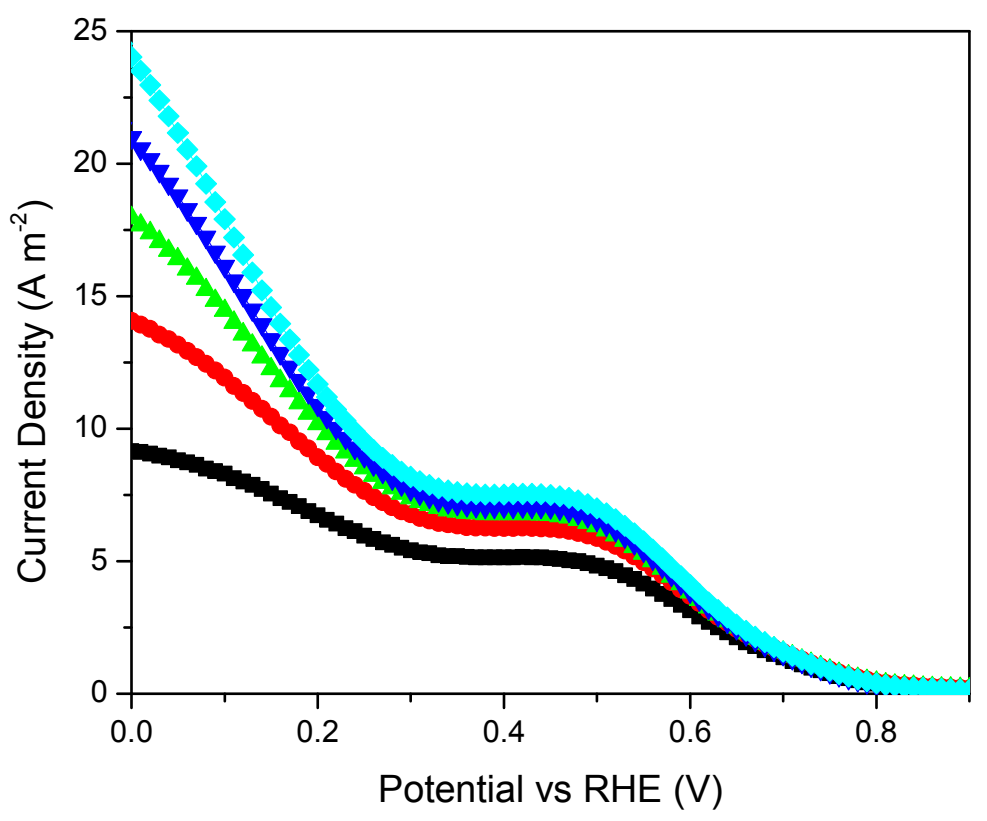

Figure S17. Ring current densities at the e-rGO electrode.

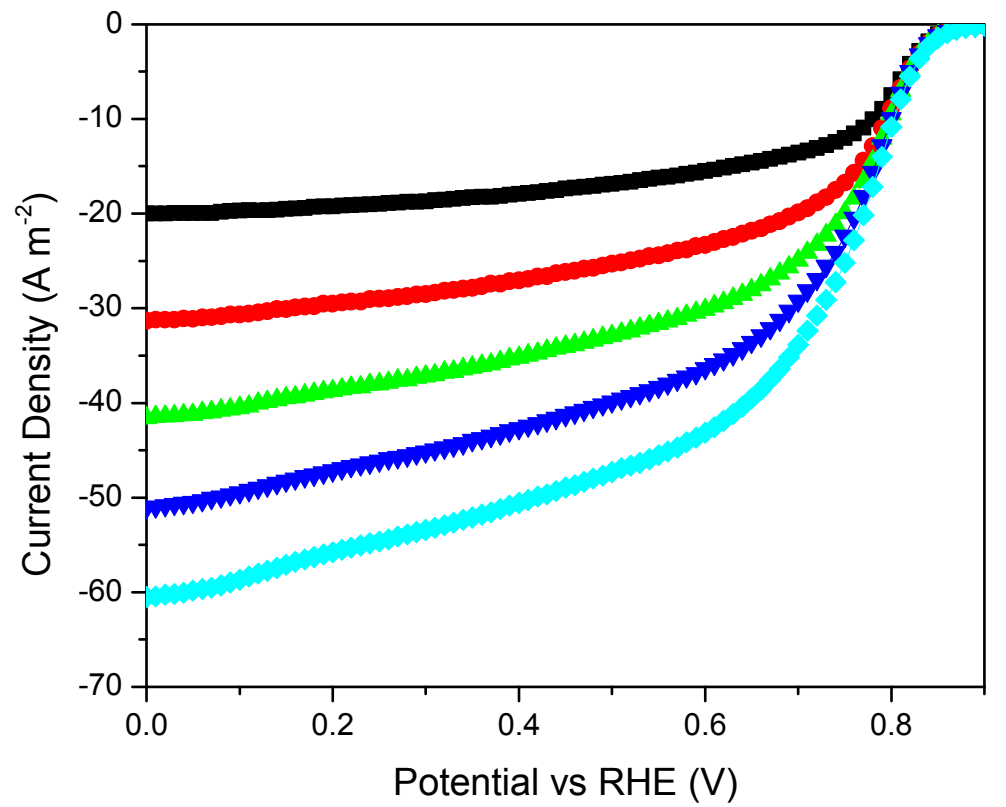

Figure S18. LSV curves for N-rGO disk. 


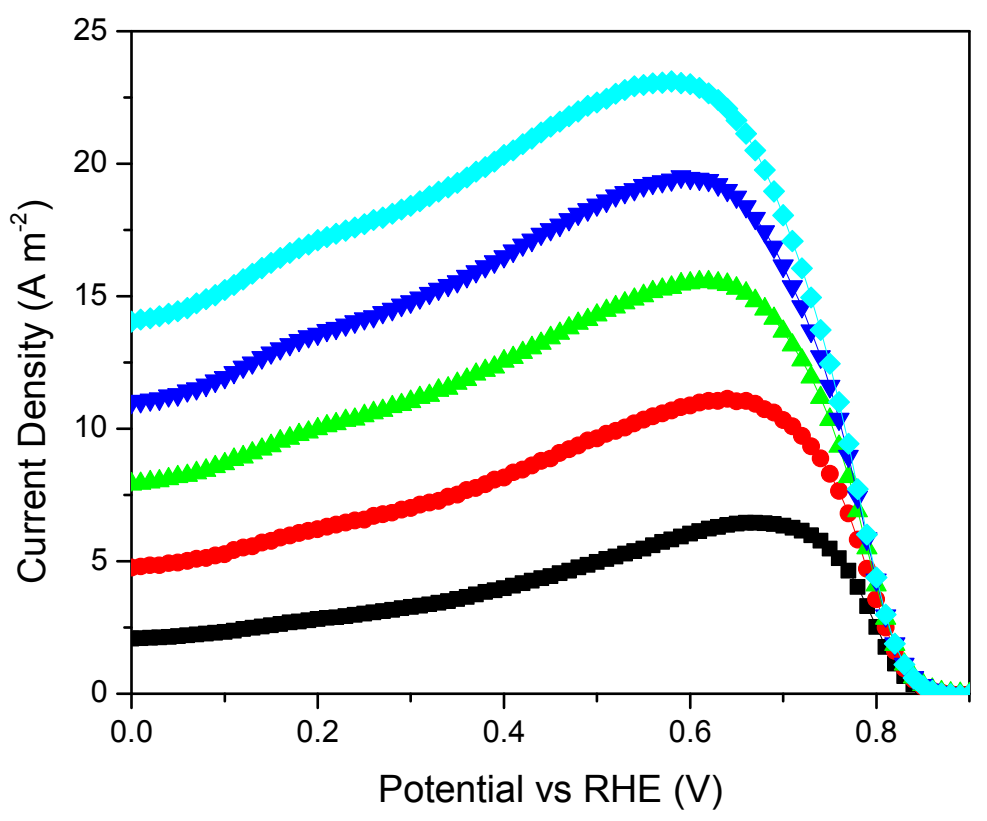

Figure S19. Ring current densities at the N-rGO electrode.

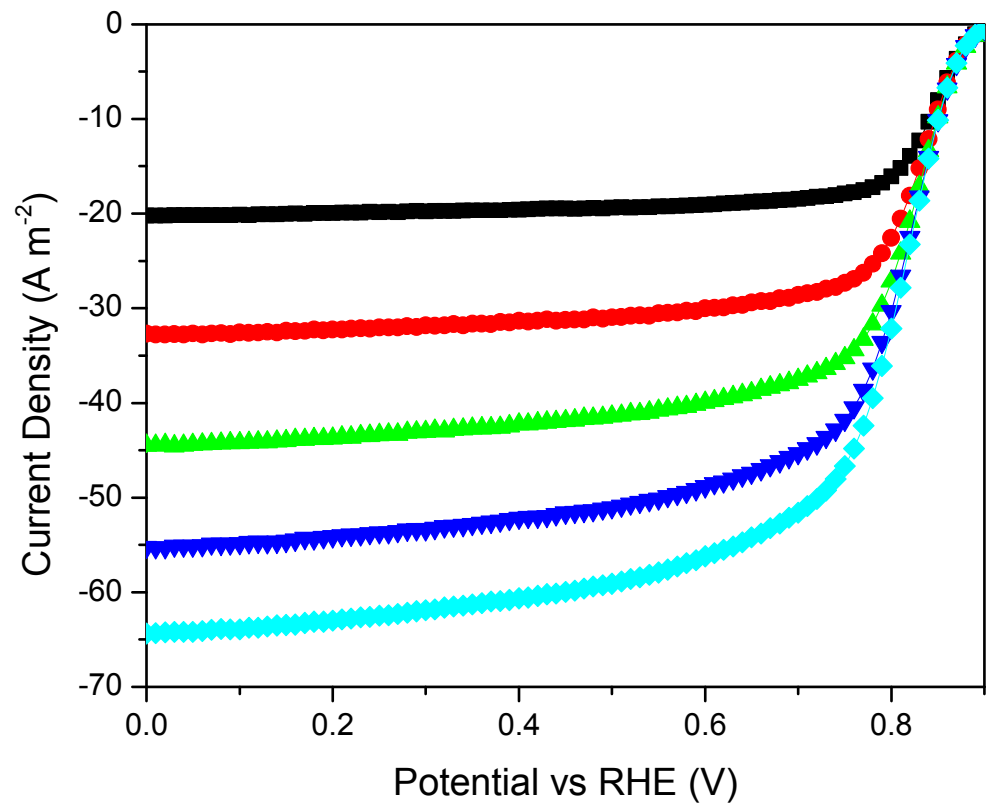

Figure S20. LSV curves for $\mathrm{Co}(\mathrm{OH})_{2} / \mathrm{N}-\mathrm{rGO}$ disk. 


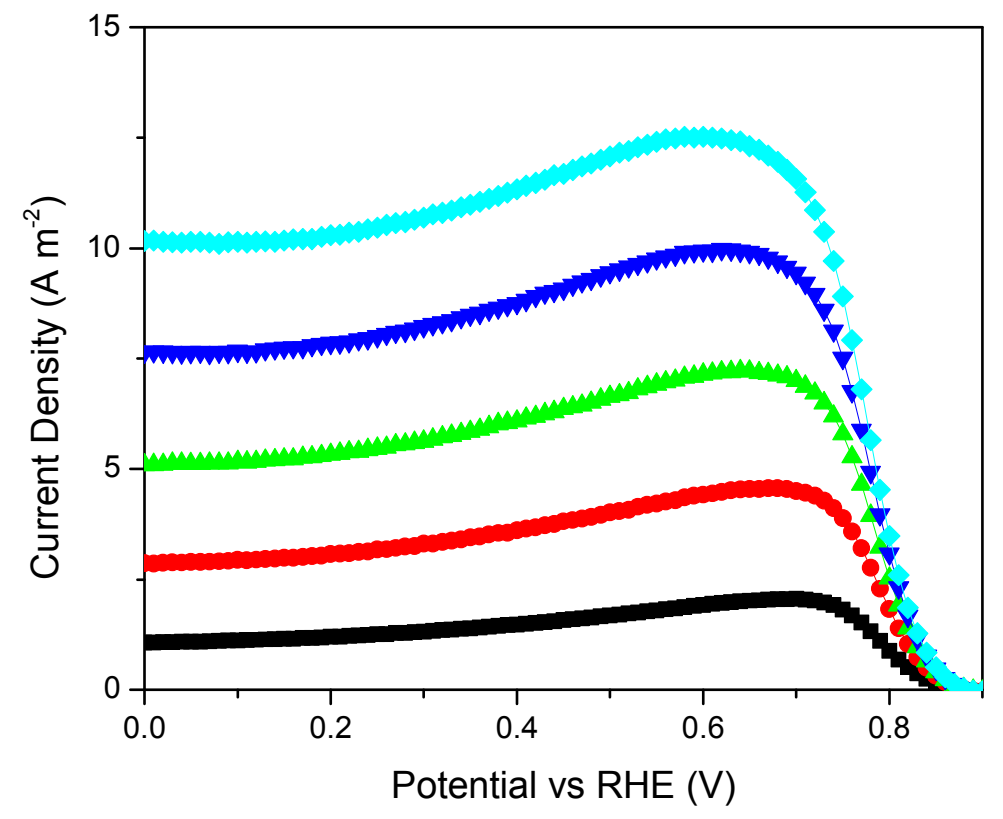

Figure S21. Ring current densities at the $\mathrm{Co}(\mathrm{OH})_{2} / \mathrm{N}-\mathrm{rGO}$ electrode.

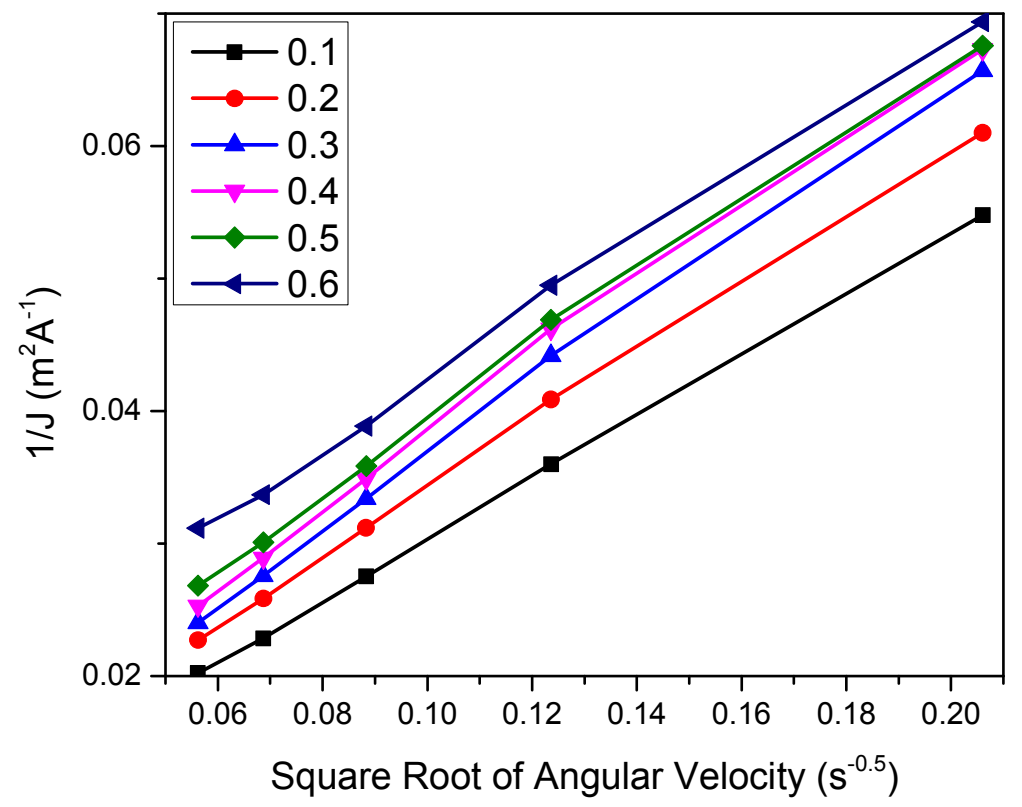

Figure S22. KL plots for Au electrode. 


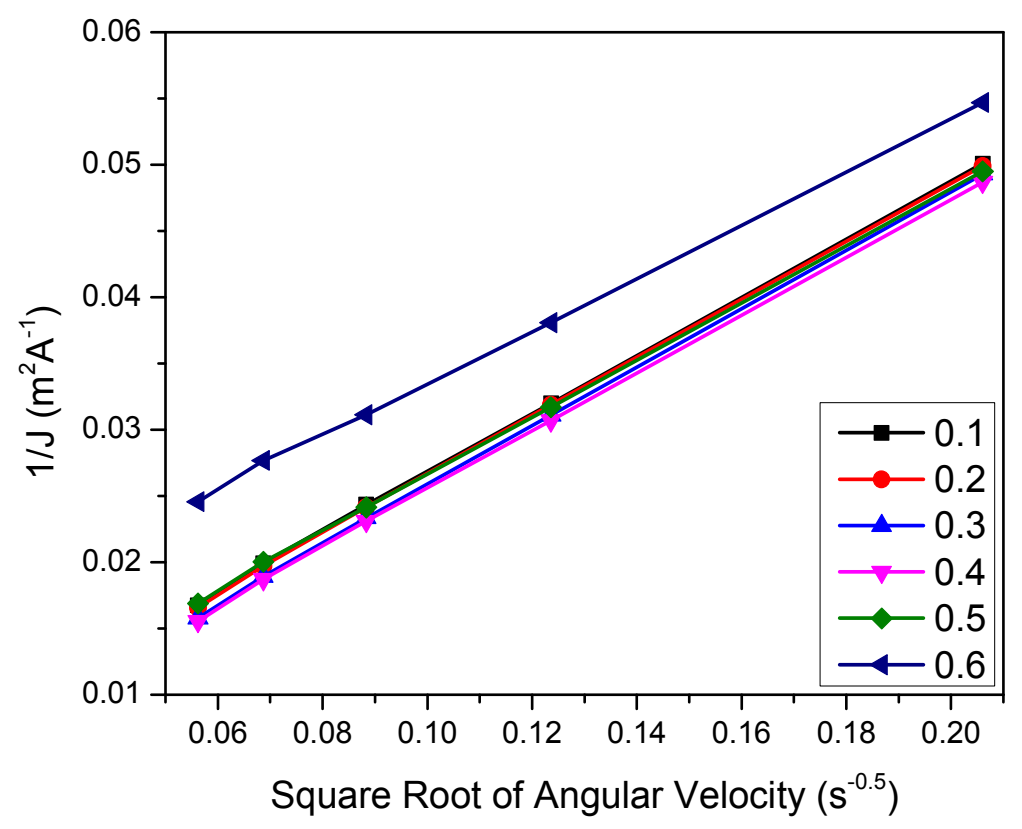

Figure S23. KL plots for Ag electrode.

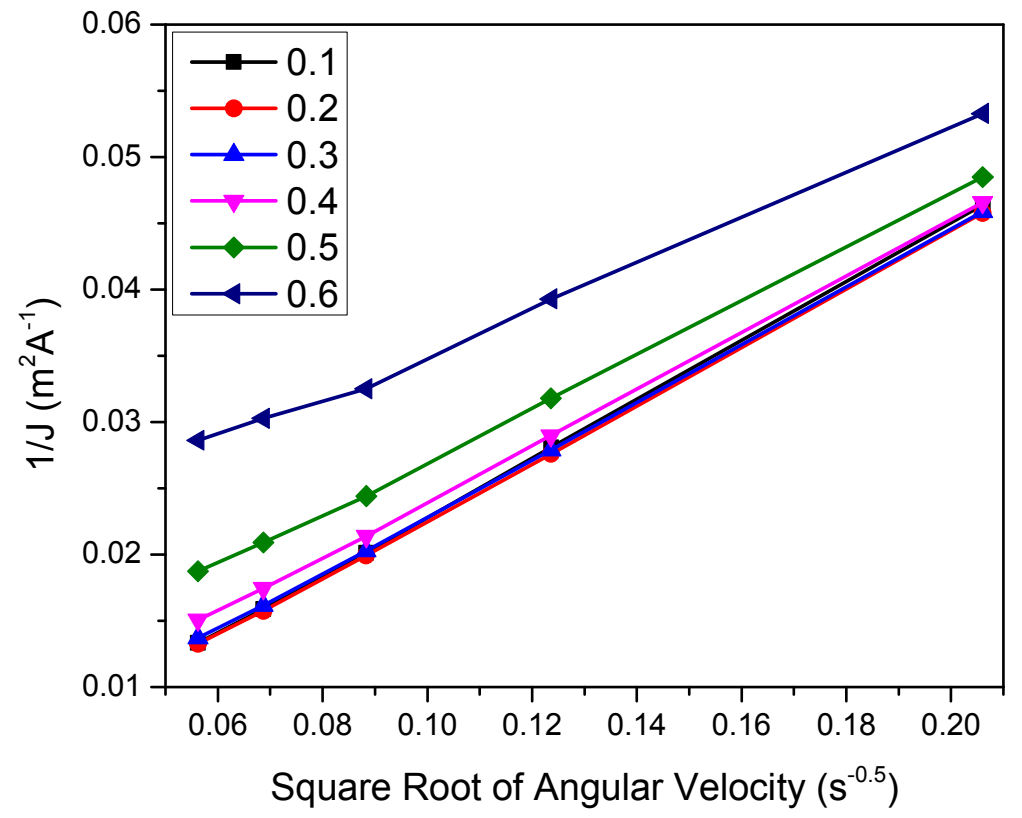

Figure S24. KL plots for Ru electrode. 


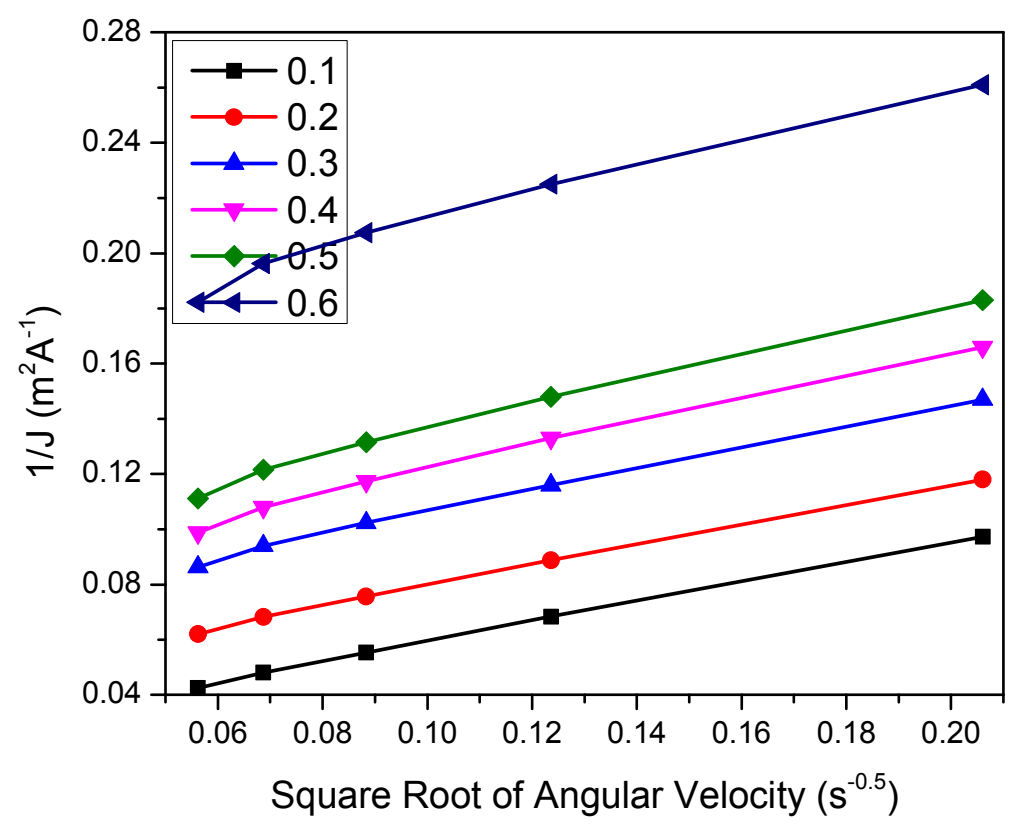

Figure S25. KL plots for e-rGO electrode.

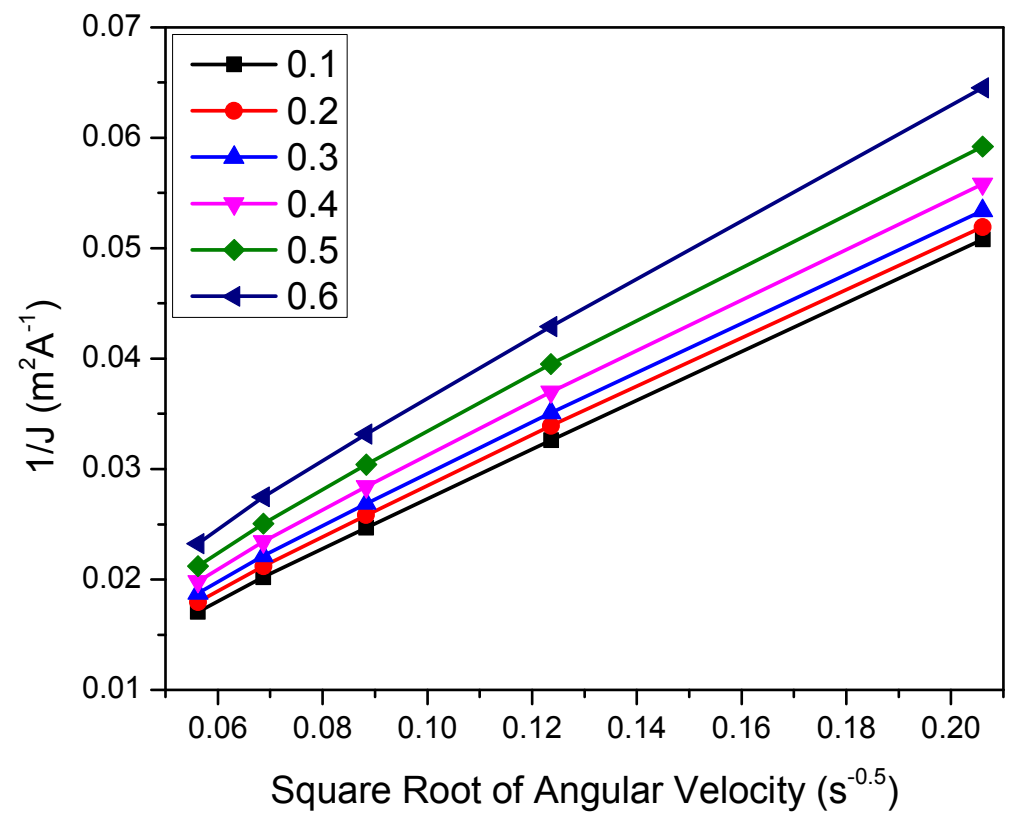

Figure S26. KL plots for N-rGO electrode. 


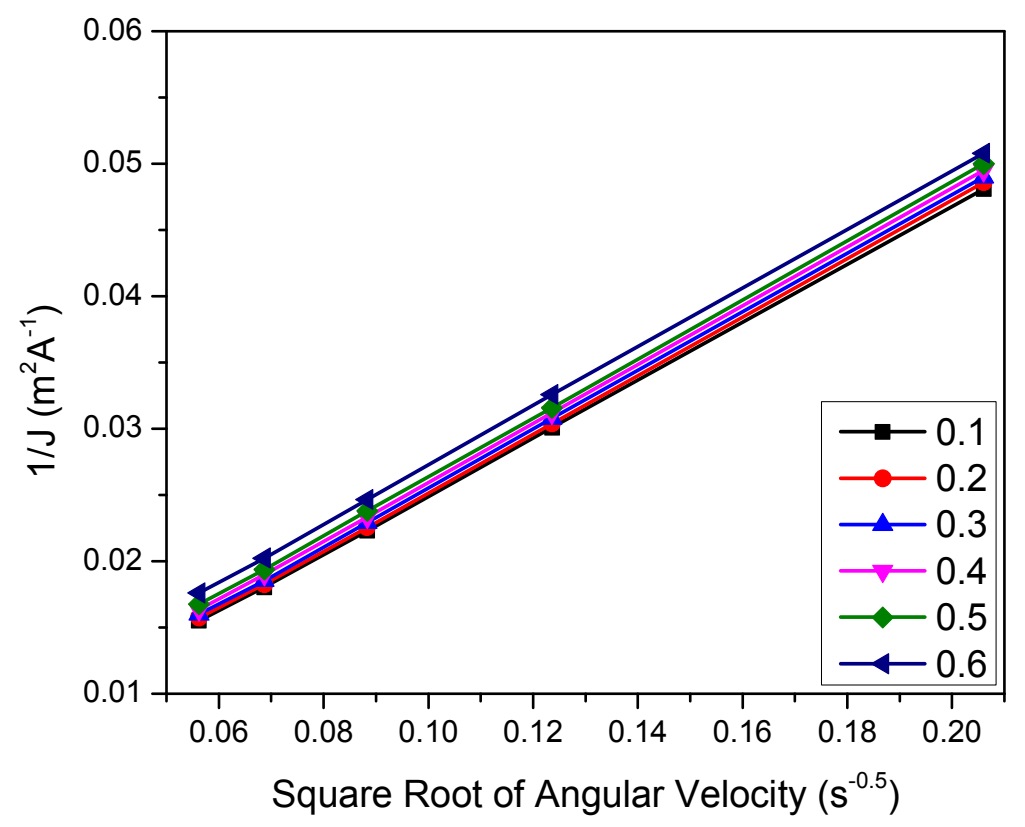

Figure S27. $\mathrm{KL}$ plots for $\mathrm{Co}(\mathrm{OH})_{2} / \mathrm{N}$-rGO electrode. 